\title{
DISCREPANCIA EN LA CLASIFICACIÓN DE COSTOS EN LA INDUSTRIA DE LA CONSTRUCCIÓN: UN ESTUDIO COMPARATIVO ENTRE EL REGLAMENTO DE LA LEY DE OBRA PÚBLICA Y SERVICIOS RELACIONADOS CON LA MISMA PARA EL ESTADO Y LOS MUNICIPIOS DE GUANAJUATO Y LAS NORMAS DE INFORMACIÓN FINANCIERA
}

Discrepancy in the classification of costs in the construction industry: A comparative study of the "Regulation of the Public Works and Related Services with the same for the state and the municipalities of Guanajuato Act" and the norms of financial reporting

Eva Lozano Montero. ${ }^{1}$

Sumario:

I. Introducción. II. Problemática. III. Metodología. IV. Conceptualización y clasificación de costos.

V. Conclusiones. VI. Bibliografía.

Resumen. En la industria de la construcción, la correcta clasificación de los costos, incluyendo los gastos directos e indirectos, es de gran importancia para dar certidumbre al proceso de toma de decisiones tanto en la determinación de la propuesta para la adjudicación de una obra, como en la utilidad esperada de la misma.

Este artículo, analiza el proceso de la clasificación de los costos y de los gastos directos e indirectos desde dos puntos de vista: el técnico, considerando el Reglamento de la Ley de Obra Pública y Servicios relacionados con la misma para el Estado y los Municipios de Guanajuato, y el contable-financiero, de acuerdo con las Normas de Información Financiera. Con base en las discrepancias de clasificación encontradas, se proponen soluciones que coadyuven a la mejora de los criterios de categorización de costos en la industria de la construcción.

Palabras clave: Clasificación de Costos, Industria de la Construcción, Ley de Obra Pública, Normas de información Financiera

Abstract. In the construction industry, the right classification of costs, including direct and indirect expenses, is of utmost importance to give certainty to the decision making process for tendering and expected profit of a given work proposal.

In this paper, the process of classification of costs and direct and indirect expenses is analyzed from two view-points: while the technical one is carried out considering the Bylaw of the Public Works and Related Services Law for the State and Municipalities of Guanajuato, the accountable and financial one is done according to the Financial Information Standards. Based on found

\footnotetext{
${ }^{1}$ Profesora de la División de Ciencias Económico Administrativas, Departamento de Gestión y Dirección de Empresas de la Universidad de Guanajuato. Maestra en Administración y Fiscal, doctorante en Ciencias de la Administración, línea de investigación en Finanzas.
} 
costs classification discrepancies, solutions to improve costs categorization for the construction industry are proposed.

Keywords: Costs Classification, Construction Industry, Public Works Law, Financial Information Standards.

\section{INTRODUCCIÓN}

Las empresas constructoras y en general cualquier persona o institución del sector de la construcción tiene como objetivo satisfacer las necesidades de los diferentes clientes a través de la prestación de un servicio de calidad, el cual tiene como resultado un producto, este producto implica en un costo de fabricación que posteriormente al colocarse en el mercado a un precio de venta razonable, cubre los costos y gastos de fabricación y además genera una utilidad deseada.

El análisis de la práctica de la integración de un presupuesto de costos, en el Estado de Guanajuato, nos muestra que una gran parte de constructores, principalmente los de micro y pequeñas empresas, definen sus precios considerando aquellos precios que genera la competencia, resultando esto en un parámetro de medición ineficiente y desconociendo si el precio fijado de tal forma, cubre la totalidad de costos generados en la empresa; la consecuencia inmediata de esta práctica es un lento desarrollo de las entidades económicas o la desaparición de las mismas. Es necesario considerar la competencia, sin olvidar la importancia del costo beneficio de la obra.

Conocer los costos en los que las empresas incurren es una de las áreas principales y clave de la correcta administración empresarial, todos los esfuerzos invertidos en la prestación de servicios y construcción, se traducen en utilidades y a su vez en desarrollo y estabilidad de la empresa.

Elaborar un presupuesto de obra, es de gran trascendencia, identificar los conceptos que son directos e indirectos pareciera ser sencillo, sin embargo; la realidad muestra que existe discrepancia en cuanto a la conceptualización de los mismos, ya que técnicamente, a partir del monto estimado de los costos directos, se establece un porcentaje de los costos indirectos para cada obra, ${ }^{2}$ los cuales, de acuerdo al procedimiento que siguen los contratistas; corresponden a los gastos generales necesarios para la ejecución de los trabajos no incluidos en los costos directos que realiza tanto en las oficinas centrales como en la obra, dichos conceptos indirectos, están representados principalmente por costos fijos que se generan en la entidad económica. Por otro lado, desde el punto de vista contable y con base en las Normas de Información Financiera (NIF), la conceptualización de costos directos e indirectos difiere, repercutiendo en la determinación de decisiones diversas por ambas metodologías.

Cualquier decisión que se considere en la organización, influye en los costos de la misma, exigiendo el suficiente análisis y garantizando en su implementación resultados favorables. Cada una de estas decisiones exige ser valuadas bajo la incidencia que tendrá sobre los costos que se tienen en determinado momento y los costos que generará la implementación de la misma.

\footnotetext{
${ }^{2}$ Periódico oficial del gobierno del Estado. Reglamento de la ley de obra pública y servicios relacionados con la misma para el Estado y los municipios de Guanajuato, Guanajuato, Gto. 2005, número 148, pp. 32-33. 
Es así como la estimación de los costos que generan las empresas es uno de los instrumentos más importantes para la toma de decisiones, y se puede decir que para evaluar los costos en los presupuestos, es necesario considerar la incidencia de cualquier decisión y las posibles eventualidades y consecuencias para la empresa.

En la industria de la construcción, existen diversos costos, que deben ser clasificados según la incidencia y relación que éstos tengan en la obra y en la empresa, además de la decisión que se desea considerar. Concretamente el análisis de la clasificación de costos estará enfocado al que establece el Reglamento de la Ley de Obra Pública y Servicios Relacionados con la misma para el Estado y los Municipios de Guanajuato y las Normas de Información Financiera.

Algunos administradores pudieran argumentar que las utilidades determinadas no concilian, debido a que el técnico realiza un Presupuesto por Obra y el contador presenta un Estado de Resultados de la empresa en un período determinado. El presupuesto de obra considera los costos y gastos necesarios para ejecutar adecuadamente la obra, aunado a los impuestos por pagar y la utilidad deseada; por lo que "debería" de coincidir con la utilidad determinada en el Estado de Resultados de la obra. Lo relevante es que al clasificar los costos, la obra debe absorber costos fijos (siendo un gran porcentaje de costos indirectos) y los variables (representados por los costos directos), pero si existieran costos fijos representativos en referencia al costo total de la obra, la decisión tomada cambiaría su rumbo, de ello dependería el porcentaje de utilidad deseada. Asimismo, debe considerarse que al no disponer de obra por ejecutar, los costos fijos los debe absorber la empresa, ocasionando disminución de utilidades y hasta pérdidas. Para comparar las utilidades obtenidas, el administrador debe elaborar un Estado de Resultados por Obra y estar en constante contacto con el responsable de la obra, independientemente de que para efectos fiscales y legales elabore un Estado de Resultados de la empresa correspondiente a un período determinado.

\section{PROBLEMÁTICA}

El técnico y el contador están preocupados por el bienestar de la empresa constructora, sin embargo; en la mayoría de las ocasiones realizan sus actividades de forma independiente, existiendo la nula comparación entre los resultados obtenidos y cooperación en la toma de decisiones, a pesar de que para un correcto análisis de costo-beneficio por obra, es relevante determinar de forma conjunta el monto de costos directos e indirectos evitando así, la fuga de recursos y la equívoca obtención de utilidad por obra.

Este problema se maximiza ya que en la clasificación de costos, comparando el criterio contable-financiero con el técnico, persiste discrepancia, provocando desentendimiento entre los profesionistas y toma de decisiones diferentes y erróneas. Lo anterior motiva a realizar un análisis de costos desde ambos puntos de vista, buscando la unificación de criterios, mejorando el entendimiento y coadyuvando a la eficiente toma de decisiones en beneficio del desarrollo y competitividad de la empresa. A continuación se dan a conocer los criterios de clasificación de cada disciplina, exponiendo fundamentos y observando cómo influyen en la determinación de la utilidad por obra.

Cada metodología clasifica los costos de obra, de acuerdo a diferentes fundamentos, lo cierto es que ambos criterios forman parte de una empresa, en donde se toman decisiones para la misma, surgiendo la preocupación de la incongruencia de clasificación de costos y las consecuencias que se desprenden, en donde el técnico presume obtener una utilidad que 
es diferente al criterio contable-financiero, generándose la incertidumbre acerca de, ¿Cuál es el criterio más razonable de clasificación de costos?

Generalmente el departamento de análisis de costos de una empresa constructora elabora el presupuesto de obra y toma decisiones sin considerar la opinión del contador o administrador. De la misma manera, el contador determina los impuestos a pagar y cargos de seguridad social, sin analizar y considerar todos los elementos que pueden contribuir en la toma de decisiones y en el desarrollo de la empresa. Este aspecto es más notorio cuando el contratista carece de un contador permanente en la empresa y solamente lo visita para motivos de cumplimiento de obligaciones fiscales y de seguridad social, sin involucrarlo en la toma de decisiones, generándose un círculo vicioso. Por lo contrario, un contador permanente, incrementa el gasto, pero el empresario le solicita mayor participación en la empresa, contribuyendo en la eficiente toma de decisiones, significando su participación e incremento en la competitividad de la empresa, de tal manera que el gasto se convierte en beneficio.

Un sistema de costos efectivo es una valiosa fuente de información estadística para el eficiente desarrollo de obras.

\section{METODOLOGÍA}

La metodología utiliza dos fases principalmente, la exploratoria y la descriptiva; donde la primera se realiza a través de una revisión de literatura relacionada con el Reglamento de la Ley de Obra Pública y Servicios Relacionados con la misma para el Estado y los Municipios de Guanajuato y con las Normas de Información Financiera.

En la segunda fase, se relata la manera de registro de los costos y sus consecuencias con las utilidades obtenidas.

\section{CONCEPTUALIZACIÓN Y CLASIFICACIÓN DE COSTOS}

\section{ASPECTO TÉCNICO}

De acuerdo a Leopoldo Varela, los cargos indirectos "son los cargos y gastos generales requeridos por la organización de campo y de la oficina central y que no pueden ser imputables en forma directa a una unidad de obra". ${ }^{3}$

Varela menciona que la Association for the Advancement of Cost Engineering (AACE), define los cargos indirectos como "todos los costos que no llegan a ser una parte final de la instalación, pero que son requeridos para ello y que pueden incluir en forma no limitativa a la administración de campo, supervisión directa, herramientas mayúsculas, costos de arranque, cuotas, seguros, impuestos, etc." 4

Por otro lado, Suárez utiliza el término de Factor de Sobre Costo, en donde incluye tanto los indirectos, como la utilidad, siendo una expresión adoptada en el Estado de Guanajuato. Define los costos indirectos como "es la suma de gastos técnico", quien a su vez define Costo Indirecto de Operación "es la suma de gastos que por su naturaleza intrínseca, son de aplicación a todas las obras efectuadas en un tiempo determinado" y costo indirecto de obra "es la

\footnotetext{
3 Varela Alonso, Leopoldo, Ingeniería de costos, teoría y práctica en construcción, México, D.F., editorial InterCost, 2009, p. 129.

4 Íbid, p. 130. 
suma de todos los gastos que por su naturaleza intrínseca, son aplicables a todos los conceptos de una obra en especial" 5

Para Suárez el costo directo "es la suma de material, mano de obra y equipo necesario para la realización de un proceso productivo", determinando a su vez, dos tipos de costos directos; Costo directo preliminar "es la suma de gastos de material, mano de obra y equipo necesario para la realización de un subproducto" y el costo directo final "es la suma de gastos de material, mano de obra, equipo y subproductos para la realización de un producto"'

Varela menciona que R.S. Means, define a los gastos indirectos o generales como "los costos de conducir un negocio, diferentes a los costos directos de la obra, incluyendo la utilidad (markup)" coincidiendo con Suárez, asimismo, Means define "markup" como "el porcentaje de otras sumas que se añade al total de todos los costos directos para determinar un precio final o alzado. En la práctica representa dos factores importantes: el primero es el costo estimado del gasto indirecto a menudo referido como gastos generales y el segundo a la utilidad prevista por el contratista"

R.S. Means conceptualiza que los indirectos incluyen la utilidad, aunque contablemente se consideran por separado, la utilidad es de naturaleza diferente a los costos indirectos y además se determina de la suma de los costos directos e indirectos, la utilidad no es directa, ni indirecta, está intrínsecamente relacionada con la obra, aunque es resultado de los costos y gastos necesarios para ejecutar la obra.

Son indirectos "los costos que no constituyen en sí mismos -no generan realidades físicaspero son indispensables para implementarla, dentro de un medio ambiente urbano o profesional, tanto por exigencias de la ciudad donde se encuentre (impuestos), como por la necesidad de protección de la sociedad (seguros y garantías), o el imperativo de que el proyecto se realice en las mejores condiciones de diseño y de construcción (honorarios). ${ }^{9}$

El artículo 33 del Reglamento de la Ley de Obra Pública y Servicios relacionados con la misma para el Estado y los Municipios de Guanajuato, cita que "conforme al artículo 59 de la Ley, el precio unitario se integra con los costos directos correspondientes al concepto de trabajo, los costos indirectos, el costo por financiamiento y el cargo por la utilidad del contratista", ${ }^{10}$ observándose que la utilidad no se encuentra inserta en los costos indirectos.

Asimismo, en el artículo 37 del mismo reglamento, se establece que "el costo directo puede ser por mano de obra, materiales, maquinaria o equipo de construcción, depreciación, inversión, seguros, mantenimiento mayor o menor, consumo, combustibles, otras fuentes de energía, lubricantes, llantas, piezas especiales, salarios de operación, herramienta de mano, equipo de seguridad y maquinaria o equipo de construcción en espera y en reserva"11

El artículo anterior, carece de una definición de costo directo que auxilie en la identificación de algún costo que no se encuentre contemplado en los conceptos citados; el Reglamento menciona ejemplos, y en los subsecuentes artículos define cada costo mencionado como directo y en otros explica la determinación del costo de cada concepto.

\footnotetext{
5 Suárez Salazar, Carlos, Costo y tiempo en edificación, $3^{\text {a }}$. Edición, México, D.F., Editorial Limusa, 2005 , p. 25.

6 Íbidem.

7 Op. cit., 3, p. 130.

8 Op. cit., 3, p. 130.

9 Arboleda López Sergio Andrés, Presupuesto y programación de obras civiles. México, D. F. Editorial Instituto Tecnológico Metropolitano, 2007, p. 84.

${ }^{10}$ Op. cit., 2, p. 11.

11 Íbid, p. 12.
} 
La Ley de Obras Públicas y Servicios relacionados con las mismas, considera en primera instancia los costos directos, los cuales a su vez están formados por costos directos de materiales, costos directos de mano de obra y costos directos de equipo y herramienta.

Varela menciona que en el documento de Terminología Estándar de la AACE, define los costos directos en dos ámbitos:

1. El de la industria de la construcción, "es el costo instalado del equipo, material y labor directamente involucrado en la construcción física de una instalación permanente", ${ }^{12} \mathrm{y}$

2. El de la industria de manufactura, servicios y otras industrias no relacionadas con el sector de la construcción, "es la porción de los costos operativos que son generalmente asignables a un producto específico o área de proceso"13

$\mathrm{Al}$ analizar la definición de costo directo que aporta la AACE, pareciera entenderse completamente los conceptos que comprende, sin embargo, al mencionar algunos ejemplos, es necesario especificar bajo qué condiciones se acepta como costo directo. Por su parte, el artículo 59 del Reglamento de la Ley de Obra Pública y Servicios Relacionados con la misma para el Estado y los Municipios de Guanajuato, menciona que "el costo indirecto corresponde a los gastos generales necesarios para la ejecución de los trabajos no incluidos en los costos directos que realiza el contratista o consultor, tanto en las oficinas centrales como en la obra y comprende entre otros: los gastos de administración, organización, dirección técnica, vigilancia, supervisión, construcción de instalaciones generales necesarias para realizar conceptos de prestaciones laborales y sociales correspondientes al personal directivo y administrativo"14

En la práctica de la presupuestación de obra, los costos indirectos se expresan como un porcentaje de los costos directos, aplicando la fórmula y realizando la operación de manera rutinaria; dejando de analizar si realmente los costos y gastos sean directos o indirectos, sin considerar que cada obra tiene sus propias características y que se debe presupuestar de acuerdo al tamaño y estructura organizacional de la empresa.

A pesar de que el artículo 60 del Reglamento mencionado, establezca que "los costos indirectos se expresarán como un porcentaje del costo directo de cada concepto de trabajo. Dicho porcentaje se calculará sumando los importes de los gastos generales que resulten aplicables y dividiendo el resultado entre el costo directo total de la obra de que se trate"15, es importante considerar que existen organizaciones en donde los gastos de oficina central representan hasta un $80 \%$ de los cargos indirectos, los cuales representan hasta un $30 \%$ de los costos directos,16por ello; el analista de costos debe concentrarse en la estructura organizacional del personal de campo y de oficina central que sea capaz de manejar la operación de la obra. Los gastos de oficina central, generalmente son costos fijos, debiendo colaborar en la eficiente coordinación de la obra, garantizando la recuperación de los mismos y determinándose no como una receta o fórmula predeterminada, sino que se debe analizar cada obra, calculando el cargo indirecto real para cada una de ellas.

Lo anterior obliga al contratista en su organización, a adoptar la flexibilidad necesaria para adecuarse tanto en aquellos casos que se le presente una demanda extraordinaria de obra, como en aquellas situaciones en donde se carece o escasea la misma. En este momento,

\footnotetext{
${ }^{12}$ Op. cit., 3, p. 81.

${ }^{13}$ Íbidem.

14 Op. cit., 2, p. 21.

${ }^{15}$ Íbidem.

${ }^{16}$ Op. cit., 3, p. 131. 
la clasificación de costos desempeña un papel importante, pues de ello depende una toma de decisiones eficiente, donde la representatividad que tienen los gastos generales respecto a los costos directos son la pauta para realizar adecuaciones en el presupuesto y para la determinación de la utilidad deseada; siendo que la utilidad se calcula con base al costo total de obra, incluyendo costos directos e indirectos. Cuando la empresa presenta gastos generales considerables respecto al costo directo de obra, obliga a realizar un análisis y ajuste de los gastos generales, más sin embargo, si la clasificación de costos es inadecuada, se estarían efectuando decisiones equívocas.

Los gastos generales que se consideran para integrar el costo indirecto y que se pueden aplicar indistintamente a la administración de oficinas centrales o a la administración de oficinas de campo, de acuerdo al artículo 61 del Reglamento de la Ley de Obra Pública y Servicios Relacionados con la misma para el Estado y los Municipios de Guanajuato, son los siguientes: ${ }^{17}$

I.- Honorarios, sueldos y prestaciones de los siguientes conceptos:

a. Personal directivo;

b. Personal técnico;

c. Personal administrativo;

d. Cuota patronal del Seguro Social y del Instituto del Fondo Nacional de la Vivienda para los Trabajadores;

e. Prestaciones a que obliga la Ley Federal del Trabajo para el personal enunciado en los incisos a, b y c de esta fracción;

f. Pasajes y viáticos del personal enunciado en los incisos a, b y c de esta fracción; y

g. Los que deriven de la suscripción de contratos de trabajo para el personal enunciado en los incisos a, b y c de esta fracción;

II.- Depreciación, mantenimiento y rentas de los siguientes conceptos:

a. Edificios y locales;

b. Locales de mantenimiento y guarda;

c. Bodegas;

d. Instalaciones generales;

e. Equipos, muebles y enseres;

f. Depreciación o renta y operación de vehículos; y

g. Campamentos;

III.- Servicios de los siguientes conceptos:

a. Consultores, asesores, servicios y laboratorios; y

b. Estudios e investigaciones;

IV.- Fletes y acarreos de los siguientes conceptos:

a. Campamentos;

b. Equipo de construcción;

$\overline{{ }^{17} \text { Op. cit., 2, pp. 21-23. }}$

CienCIA JuRídicA. Departamento de Derecho. División de Derecho Política y Gobierno, Universidad de Guanajuato - Año 1, No. 2, 2012 
c. Plantas y elementos para instalaciones; y

d. Mobiliario;

V.- Gastos de oficina de los siguientes conceptos:

a. Papelería y útiles de escritorio;

b. Correos, fax, teléfonos, telégrafos y radio;

c. Equipos de cómputo;

d. Situación de fondos;

e. Copias y duplicados;

f. Luz, gas y otros consumos; $y$

g. Gastos de la licitación;

VI.- Capacitación y adiestramiento;

VII.- Seguridad e higiene;

VIII.- Seguros y fianzas; y

IX.- Trabajos previos y auxiliares de los siguientes conceptos:

a. Construcción y conservación de caminos de acceso;

b. Montajes y desmantelamiento de equipo;

c. Señalamiento indicativo y protección de obra; y

d. Construcción de instalaciones generales:

1. De campamentos;

2. De equipo de construcción; y

3. De plantas y elementos para instalaciones.

Con relación a los costos indirectos, el artículo 61 del RLOPEG considera que son aquellos generados por el personal administrativo y que no interviene directamente en la ejecución de los trabajos, sino que son inherentes a estos; sin importar si son de la administración de las oficinas centrales o de las oficinas de campo, sin embargo, desde la perspectiva contablefinanciera, esto es de gran importancia para considerarlo como directo o indirecto, ya que cada costo puede ser directo o indirecto respecto a su identificación y cuantificación en este caso con la obra a ejecutar, así como el origen del mismo.

Antes de realizar el análisis comparativo entre ambas disciplinas, es necesario definir la forma de determinar el precio unitario de cada concepto de trabajo en un presupuesto de obra. Cuando el especialista en costos de la Industria de la Construcción, determina el precio unitario de cada concepto de trabajo, se sabe que dicho precio está integrado por los siguientes conceptos:

a. Costos directos correspondientes al concepto de trabajo, que considera los insumos necesarios para la ejecución de la obra.

b. ya que corresponden a los gastos generales necesarios para la ejecución de los trabajos no incluidos en los costos directos que realiza el contratista, tanto los de las oficinas centrales como los del sitio de la obra. 
c. Costo por financiamiento.- Este costo se estima como un porcentaje de la suma de los costos directos e indirectos, y representa los gastos generados de la inversión de los recursos propios de la entidad económica o recursos ajenos que efectúe el contratista en cumplimiento a la ejecución de la obra.

d. Utilidad deseada.- Es la ganancia que el contratista desea recibir por los trabajos realizados, en donde cada contratista fija el porcentaje de utilidad. El porcentaje de utilidad es sobre el costo total de fabricación (costos directos + costos indirectos + costos de financiamiento).

e. Cargos adicionales.- Son erogaciones que realiza el contratista, siendo obligaciones adicionales que derivan de algún impuesto y no están considerados en los apartados anteriores. $^{18}$

Aunado a lo anterior, la Secretaría de Obra Pública del Estado de Guanajuato, tiene establecido un formato en donde se especifican los conceptos de costos directos e indirectos que se manejan en los presupuestos de obra, fundamentado en el Reglamento de la Ley de Obra Pública en referencia. Sin embargo ¿cuál es el fundamento conceptual para establecer algunos conceptos como directos y otros como indirectos? ¿Es un fundamento lógico y razonable la clasificación que se establece? ¿La clasificación es aplicable para todas las obras que se presupuestan?

Se hace referencia al Capítulo Sexto del Reglamento de la Ley de Obras Públicas y Servicios relacionados con las mismas, (RLOPF), en donde en la Sección II, a partir del artículo 190, explica la determinación de los conceptos que se incluyen como costos directos, sin mencionar qué es un costo directo. En la sección III, artículo 211, menciona que el "costo indirecto corresponde a los gastos generales necesarios para la ejecución de los trabajos no incluidos en los costos directos que realiza el contratista, tanto en sus oficinas centrales, como en la obra, ...", ${ }^{19}$ coincidiendo los conceptos con los mencionados en el Reglamento de la Ley de Obra Pública y Servicios Relacionados con la misma para el Estado y los Municipios de Guanajuato, así mismo; en el artículo 212, se menciona que los costos indirectos se expresarán como un porcentaje del costo directo de cada concepto de trabajo, estando en el mismo sentido las leyes y reglamentos federales y estatales. ${ }^{20}$ Otros autores del área de Ingeniería de Costos, coinciden con las definiciones y elementos que se consideran en cada clasificación.

Sin embargo, al observar los conceptos que se contemplan en los costos directos e indirectos de acuerdo al Reglamento de la Ley de Obra Pública y Servicios Relacionados con la misma para el Estado y los Municipios de Guanajuato, se pueden determinar diferencias de clasificación con los criterios contable - financiero establecido tanto en las NIF (Normas de Información Financiera) como en algunos textos contables.

En las Normas de Información Financiera; Boletín D-7, se definen entre otros conceptos, lo que es un contrato de construcción y los tipos de contratos de obra; también incluye algunas reglas de valuación y los axiomas referentes a qué es un costo directo e indirecto,

\footnotetext{
${ }^{18}$ González Meléndez, Raúl, "Unificando Criterios”, Ingeniería Financiera y de Costos, México, D.F. año LIX, núm., 484, agosto de 2009, pp. 28-32.

19 Diario Oficial de la Federación. Reglamento de la Ley de Obras Públicas y Servicios Relacionados con las mismas, 2010, p. 72.

${ }^{20}$ Op. cit., 19, p. 72.
} 
específicamente en los párrafos 32 a 37, acotando las actividades más comunes para cada clasificación.

Son costos directos, los que "se relacionan directamente con el contrato específico" y los costos indirectos son aquellos que "son atribuibles a la actividad general del contratista y que puedan ser asignados a un contrato específico". ${ }^{21}$

Asimismo, las NIF dan a conocer los costos directos: ${ }^{22}$

a. Mano de Obra en el sitio de la construcción, incluyendo supervisión

b. Materiales usados en la construcción

c. Adquisición propiedades, planta y equipo destinados exclusivamente al contrato

d. Depreciación de propiedades, planta y equipo usados en la obra

e. Traslado de maquinaria, equipo y materiales hacia y del sitio de la construcción

f. Rentas de propiedades, planta y equipo

g. Diseño y asistencia técnica

h. Costos estimados de rectificación de especificaciones originales y trabajo de garantía, incluyendo los costos de garantía esperados

i. Reclamaciones de terceras partes

j. Seguros y fianzas relacionados con la construcción

k. Costo o resultado integral de financiamiento directamente atribuible a los financiamientos obtenidos para el desarrollo del contrato, atendiendo las disposiciones vigentes.

Se consideran dentro de los Costos Indirectos, los siguientes conceptos:

a. Seguro y fianzas generales

b. Diseño y asistencia técnica que no estén relacionados con un contrato específico

c. Mano de obra indirecta

d. Supervisión indirecta del contrato

e. Suministros

f. Herramientas y equipo

g. Control de calidad e inspección

\footnotetext{
${ }^{21}$ Normas de información financiera, sexta edición, México, D.F. Instituto Mexicano de Contadores Públicos, 2011, p. 717.

${ }^{22}$ Íbid, pp. 717-718.

Ciencia JuRídicA. Departamento de Derecho. División de Derecho Política y Gobierno, Universidad de Guanajuato - Año 1, No. 2, 2012
} 


\section{h. Reparación y mantenimiento}

i. En algunas circunstancias, costos base como la preparación y procesamiento central de nóminas. ${ }^{23}$

Dichos costos son distribuidos entre todos los contratos usando métodos sistemáticos y racionales, aplicados consistentemente.

Los costos iniciales deben incluirse como costos del contrato, considerando que son aquellos costos incurridos una vez que se ha adjudicado el contrato de construcción y antes del inicio de las actividades propias del mismo, como pueden ser los costos por instalaciones de campamentos, construcción de caminos de acceso, instalación de plantas y otros.

Mientras que los costos erogados que están directamente relacionados con el contrato, cuya recuperación está implícita en el presupuesto de obra original, como los costos de ingeniería básica y otros costos fijos, en caso de que se gane el concurso de obra, los debe absorber el contrato; por el contrario, en caso de perder el concurso o que los costos tengan alta posibilidad de no ser recuperados, o que no puedan ser identificados y cuantificados por separado, deben incluirse como gastos del período.

De igual forma, en las NIF, Boletín D-7, párrafo 40, se especifica que los costos que no pueden ser atribuibles directamente al contrato, o no pueden ser asignados a contratos específicos, o cuyo cobro no esté especificado en el contrato, deben excluirse y por tanto reconocerse en los gastos del período y no del producto. Entre tales costos a excluir estarían: ${ }^{24}$

a. Los gastos generales de administración, para los que no se haya especificado ningún tipo de reembolso en el contrato.

b. Los gastos de venta.

c. Los gastos de investigación y desarrollo para los que, en el contrato, no se especifica reembolso alguno.

d. La parte de depreciación correspondiente a propiedades, planta y equipo que no han sido utilizados en algún contrato específico.

Lo anterior no significa que dichas erogaciones sean ignoradas en la determinación del costo total, contablemente se consideran gastos del período y no costos del producto, aunque sí forman parte del costo total.

Asimismo, existen autores del área contable financiera que conceptualizan los costos. Para García, ${ }^{25}$ los costos directos "Son aquellos costos que se pueden identificar o cuantificar plenamente con los productos terminados o áreas específicas”; Cárdenas, ${ }^{26}$ considera que “... podría suceder que un gasto fuese causado por un producto particular y se puede cargar directamente al costo de ese producto..."; coincidiendo con la siguiente definición de Ramírez27,

${ }^{23}$ Íbid, p. 718.

24 Íbidem.

${ }^{25}$ García Colín, Juan, Contabilidad de costos 2, segunda edición, México, D. F., ed. Mc Graw Hill, 2001, p. 13.

${ }^{26}$ Cárdenas Nápoles, Raúl, Contabilidad de costos 1, tercera edición, México, D.F., ed. ANFECA, IMCP, 1999, p. 39.

${ }^{27}$ Ramírez Padilla, David Noel, Contabilidad administrativa, octava edición, México, D.F., ed. McGraw Hill, 2008, p. 37 . 
"son los que se identifican plenamente con una actividad, departamento o producto."Por otra parte, los Costos Indirectos, son conceptualizados por cada autor, de tal manera que coinciden en lo que se refiere a que "son aquellos costos que no se pueden identificar o cuantificar plenamente con los productos terminados o áreas específicas."; 28 "son los que se efectúan en la ejecución de los actos generales de la empresa, precisamente relacionados con la producción, ..."; ;9 y a que "es el que no se puede identificar con una actividad determinada." 30

En resumen, las características de los cargos indirectos se pueden agrupar en los siguientes:

1. Los cargos indirectos involucran una serie de conceptos diferentes (como agua, luz, gasolina, entre otros), que no corresponden a la materia prima directa y a la mano de obra directa.

2. Estos costos tienen en común la falta de identificación y cuantificación con el departamento y principalmente con el producto, por lo que se hace compleja su integración al costo de producción.

3. Por lo anterior, es imposible determinar el costo unitario rigurosamente exacto, aun cuando se utilicen métodos que ya se han desarrollado, cuya finalidad es prorratear los costos de una manera adecuada y eficaz.

Aparentemente, coinciden las definiciones entre las disciplinas de la construcción (Ingeniería de Costos), con la contable financiera (Contabilidad de Costos), pero al momento de catalogar los conceptos de obra, es notoria la discrepancia de clasificación; en la Tabla número 1, se realiza un análisis de cada concepto, considerando como base, la clasificación técnica, realizando una comparativa con observaciones en cada uno de los conceptos.

Existen aproximadamente veinte y dos conceptos que requieren de un análisis profundo de su incidencia en el contrato de obra, exigiendo la unificación de criterios tanto en el aspecto técnico como contable financiero, coadyuvando a la toma de decisiones que repercutirá en el beneficio esperado para la organización. Es imperativo la homologación de conceptos entre los emisores de la normatividad, exhortando a la Comisión de Obra Pública del Congreso, con el Consejo Mexicano para la Investigación y Desarrollo de Normas de Información Financiera (CINIF) y el Instituto Mexicano de Contadores Públicos (IMCP), que en coordinación con colegios y cámaras del sector de la construcción y colegios de contadores públicos reforzarán y retroalimentarán los criterios establecidos, de donde se desprenderán nuevas reglas necesarias para el eficiente desarrollo y competitividad de las empresas constructoras. Con lo anterior, las Normas de Información Financiera y el reglamento de la Ley de Obra Pública y Servicios relacionados con la misma para el estado y los municipios de Guanajuato presentarán conjuntamente clasificaciones lógicas, razonables y justificables para el sector de la construcción.

Toda empresa constructora como sociedad mercantil persigue el lucro, por lo que la utilidad neta es, desde el punto de vista contable, el valor residual de los ingresos de una entidad lucrativa después de haber disminuido sus costos y gastos relativos, así como el impuesto sobre la renta (ISR) y la participación de las utilidades a los trabajadores (PTU) correspon-

\footnotetext{
${ }^{28}$ Op. cit., 25, p. 13.

29 Op. cit., 26, p. 40.

${ }^{30}$ Op. cit., 27, p. 38. 
dientes; presentados en el estado de resultados, durante un periodo contable; siempre que éstos últimos sean menores a dichos ingresos. En caso contrario, cuando los costos y gastos son superiores a los ingresos, la resultante es una pérdida del ejercicio.

La forma de obtener la utilidad en un Estado de Resultados es la siguiente:

Estado de Resultados del al de

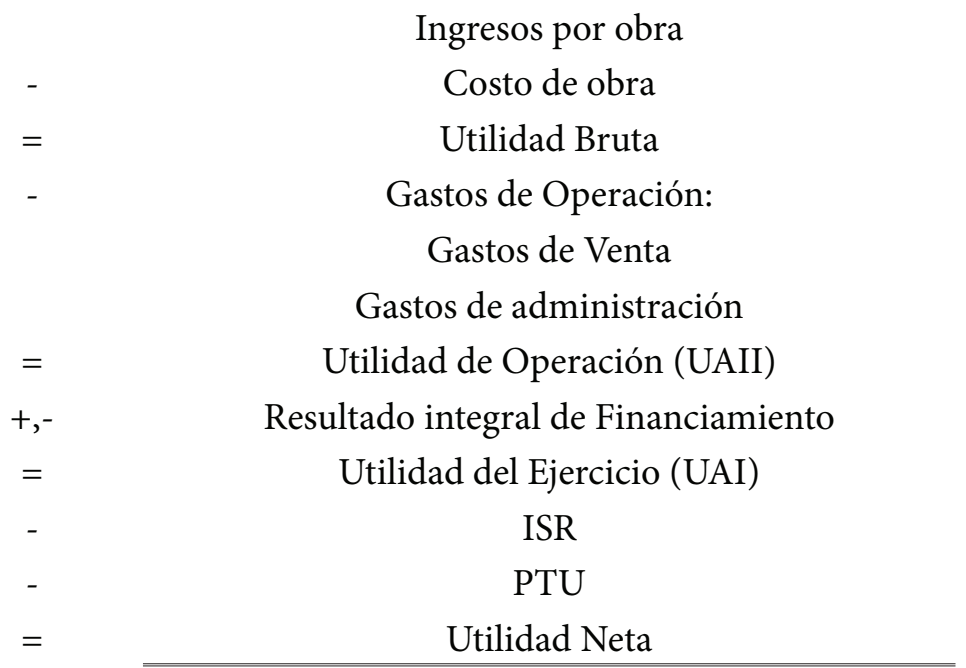

Se propone un Estado de Resultados por Obra, para estar en condiciones de compararlo con el Proyecto de Obra y se facilite la comparación de resultados, asimismo; se podrán tomar decisiones relativas a exceso de costos o gastos generales, involucrando al constructor en el análisis de costos en beneficio de la empresa.

A continuación, se esquematiza la metodología de determinación de la utilidad, considerando el enfoque de la disciplina de Ingeniería de Costos.

$\begin{array}{cc} & \text { Costos Directos } \\ + & \text { Costos Indirectos } \\ & \text { Costos por financiamiento } \\ & \text { Costos Adicionales } \\ + & \text { Costo Total } \\ = & \text { Utilidad deseada } \\ & \text { Precio unitario o alzado }\end{array}$

Cabe señalar que en los costos directos e indirectos, se incluyen elementos que contablemente se conocen como gastos de operación y los de financiamiento, asimismo, incluye el ISR y el PTU. Lo anterior significa que se obtendrían utilidades muy similares por ambas metodologías, aunque la preocupación radica en la toma de decisiones al momento de clasificar los costos; en donde se propone analizar la naturaleza de cada uno de ellos y que no solamente se apliquen fórmulas preestablecidas, que se considere la flexibilidad de los gastos generales 
y en donde los contadores o administradores elaboren Estados de Resultados por Obra y aporten reflexiones del análisis de costos, provocando reacciones en la utilidad deseada.

Al comparar un Estado de Resultados de una obra en específico elaborado por el administrador, con el Presupuesto de Obra realizado por el técnico (considerando las modificaciones autorizadas, como conceptos fuera de catálogo y escalatorias), obtendríamos diferentes utilidades, debido principalmente a la clasificación de costos y a la carencia de comunicación entre ambas partes.

Tabla número 1. Análisis de clasificación de conceptos en la determinación de costos.

\begin{tabular}{|c|c|c|c|}
\hline $\begin{array}{c}\text { Costo de obra } \\
\text { Concepto }\end{array}$ & $\begin{array}{c}\text { Reglamento } \\
\text { de la lop }\end{array}$ & Nif(boletin d-7) & Observaciones \\
\hline Mano deObra & Directo & Directo & \\
\hline Materiales & Directo & Directo & \\
\hline $\begin{array}{l}\text { Maquinaria y equipo de } \\
\text { construcción (uso) }\end{array}$ & Directo & Directo & $\begin{array}{l}\text { Que sea para el contra- } \\
\text { to en específico }\end{array}$ \\
\hline Depreciación & Directo & Directo & $\begin{array}{l}\text { Cuantificable para el } \\
\text { contrato en específico }\end{array}$ \\
\hline Inversiones & Directo & Directo & $\begin{array}{l}\text { Cuantificable para el } \\
\text { contrato en específico. }\end{array}$ \\
\hline Seguros & Directo & Directo & $\begin{array}{l}\text { Cuantificable para el } \\
\text { contrato en específico }\end{array}$ \\
\hline $\begin{array}{l}\text { Mantenimiento mayor o } \\
\text { menor }\end{array}$ & Directo & Directo & $\begin{array}{l}\text { Si se calcula por obra } \\
\text { y que sea cuantificable } \\
\text { para el contrato en es- } \\
\text { pecífico. }\end{array}$ \\
\hline Combustibles & Directo & Directo o indirecto & $\begin{array}{l}\text { Cuantificable para el } \\
\text { contrato en específico }\end{array}$ \\
\hline Otras fuentes de energía & Directo & Directo & \\
\hline Lubricantes & Directo & Directo o indirecto & $\begin{array}{l}\text { Cuantificable para el } \\
\text { contrato en específico }\end{array}$ \\
\hline Llantas & Directo & Directo o indirecto & $\begin{array}{l}\text { Cuantificable para el } \\
\text { contrato en específico }\end{array}$ \\
\hline Piezas especiales & Directo & Directo & \\
\hline $\begin{array}{l}\text { Salarios de operación } \\
\text { (personal de operación de } \\
\text { máquinas) }\end{array}$ & Directo & Directo o indirecto & $\begin{array}{l}\text { Que se pueda cuantifi- } \\
\text { car para el contrato en } \\
\text { específico }\end{array}$ \\
\hline Herramienta de mano & Directo & Directo & \\
\hline $\begin{array}{l}\text { Equipo de seguridad (para } \\
\text { protección del trabajador) }\end{array}$ & Directo & Directo & \\
\hline
\end{tabular}




\begin{tabular}{|c|c|c|c|}
\hline $\begin{array}{l}\text { Maquinaria o equipo de } \\
\text { construcción en espera y } \\
\text { en reserva (erogaciones } \\
\text { derivadas de situaciones } \\
\text { no previstas) }\end{array}$ & Directo & Directo & \\
\hline $\begin{array}{l}\text { I. HONORARIOS, suel- } \\
\text { dos y prestaciones de: } \\
\text { Personal Directivo, técni- } \\
\text { co y administrativo. Se- } \\
\text { guro Social, INFONAVIT, } \\
\text { prestaciones, pasajes y } \\
\text { viáticos del personal men- } \\
\text { cionado. }\end{array}$ & Indirecto & $\begin{array}{l}\text { Indirecto o gastos } \\
\text { del período }\end{array}$ & $\begin{array}{l}\text { Observar párrafos 38, } \\
39 y 40 \text { de NIF }\end{array}$ \\
\hline $\begin{array}{l}\text { I I.DE P R E C I A C I Ó N, } \\
\text { MANTENIMIENTO Y } \\
\text { RENTAS DE: }\end{array}$ & & & \\
\hline Edificios y locales & Indirecto & Indirecto & \\
\hline $\begin{array}{l}\text { Locales de mantenimiento } \\
\text { y guarda }\end{array}$ & Indirecto & Directo o indirecto & $\begin{array}{l}\text { Si es del contrato, es di- } \\
\text { recto }\end{array}$ \\
\hline Bodegas & Indirecto & Directo a indirecto & $\begin{array}{l}\text { Si es para el contrato de } \\
\text { obra, es directo }\end{array}$ \\
\hline Instalaciones generales & Indirecto & Indirecto & \\
\hline $\begin{array}{l}\text { Equipos, muebles y ense- } \\
\text { res }\end{array}$ & Indirecto & Indirecto & \\
\hline $\begin{array}{l}\text { Depreciación o renta y } \\
\text { operación de vehículos }\end{array}$ & Indirecto & Directo o indirecto & $\begin{array}{l}\text { Directo, sólo si es para } \\
\text { el contrato de obra. }\end{array}$ \\
\hline Campamentos & Indirecto & $\begin{array}{l}\text { Directo, general- } \\
\text { mente los campa- } \\
\text { mentos son para un } \\
\text { contrato. }\end{array}$ & $\begin{array}{l}\text { Identificable solamente } \\
\text { con el contrato, es di- } \\
\text { recto. }\end{array}$ \\
\hline $\begin{array}{l}\text { III. SERVICIOS de con- } \\
\text { sultores, asesores, servi- } \\
\text { cios y laboratorios; }\end{array}$ & Indirecto & Directo e indirecto & $\begin{array}{l}\text { Los servicios de labo- } \\
\text { ratorio son para una } \\
\text { obra, siendo Directo. } \\
\end{array}$ \\
\hline $\begin{array}{l}\text { Servicios de estudios e in- } \\
\text { vestigaciones. }\end{array}$ & Indirecto & Indirecto & \\
\hline
\end{tabular}




\begin{tabular}{|c|c|c|c|}
\hline $\begin{array}{l}\text { IV. FLETES Y ACA- } \\
\text { RREOS DE: Campamen- } \\
\text { tos, }\end{array}$ & Indirecto & $\begin{array}{l}\text { Directo, los cam- } \\
\text { pamentos son para } \\
\text { contratos en espe- } \\
\text { cífico. }\end{array}$ & $\begin{array}{l}\text { Si es para el contrato de } \\
\text { obra, es directo }\end{array}$ \\
\hline Equipo de construcción, & Indirecto & Directo o indirecto & $\begin{array}{l}\text { Si es para el contrato de } \\
\text { obra, es directo }\end{array}$ \\
\hline $\begin{array}{l}\text { Plantas y elementos para } \\
\text { instalaciones }\end{array}$ & Indirecto & Directo o indirecto & $\begin{array}{l}\text { Directo, si se identifica } \\
\text { plenamente con el con- } \\
\text { trato de obra. }\end{array}$ \\
\hline Mobiliario & Indirecto & Directo o indirecto & $\begin{array}{l}\text { Si es para el contrato de } \\
\text { obra, es directo }\end{array}$ \\
\hline $\begin{array}{l}\text { V. GASTOS DE OFICINA } \\
\text { DE: Papelería y útiles de } \\
\text { escritorio; Correos, fax, } \\
\text { teléfonos, telégrafos y } \\
\text { radio; Equipos de cómpu- } \\
\text { to; Situación de fondos; } \\
\text { Copias y duplicados; Luz, } \\
\text { gas y otros consumos; y } \\
\text { Gastos de licitación; }\end{array}$ & Indirecto & Indirectos & $\begin{array}{l}\text { Observar párrafos 38, } \\
39 \text { y } 40 \text { de NIF. }\end{array}$ \\
\hline $\begin{array}{l}\text { VI. Capacitación y adies- } \\
\text { tramiento }\end{array}$ & Indirecto & Directo o indirecto & $\begin{array}{l}\text { Si es para personal de } \\
\text { la obra en específico, es } \\
\text { directo. }\end{array}$ \\
\hline VII. Seguridad e Higiene & Indirecto & Directo o indirecto & $\begin{array}{l}\text { Si se identifica plena- } \\
\text { mente con el contrato } \\
\text { de obra, es directo } \\
\end{array}$ \\
\hline VIII. Seguros y Fianzas & Indirecto & Directo & $\begin{array}{l}\text { Las fianzas son por } \\
\text { contrato. }\end{array}$ \\
\hline $\begin{array}{l}\text { IX. TRABAJOS PREVIOS } \\
\text { Y AUXILIARES DE: } \\
\text { Construcción y conser- } \\
\text { vación de caminos de } \\
\text { acceso. }\end{array}$ & Indirecto & Directo & $\begin{array}{l}\text { Si es para el contrato } \\
\text { de obra, es directo. En } \\
\text { NIF se llaman costos } \\
\text { iniciales y los absorbe } \\
\text { la obra. }\end{array}$ \\
\hline $\begin{array}{l}\text { Montajes y desmantela- } \\
\text { miento de equipo; }\end{array}$ & Indirecto & Directo & $\begin{array}{l}\text { Si es para el contrato de } \\
\text { obra, es directo }\end{array}$ \\
\hline
\end{tabular}




\begin{tabular}{|l|l|l|l|}
\hline $\begin{array}{l}\text { Señalamiento indicativo y } \\
\text { protección de obra; }\end{array}$ & Indirecto & Directo & $\begin{array}{l}\text { Generalmente es para } \\
\text { contrato de obra espe- } \\
\text { cífico y es directo. }\end{array}$ \\
\hline $\begin{array}{l}\text { Construcción de insta- } \\
\text { laciones generales de: } \\
\text { campamentos; de equipo } \\
\text { de construcción; y de } \\
\text { plantas y elementos para } \\
\text { instalaciones. }\end{array}$ & Indirecto & Directo & $\begin{array}{l}\text { Generalmente es para } \\
\text { contrato de obra en es- } \\
\text { pecífico y es directo. }\end{array}$ \\
\hline
\end{tabular}

Fuente Propia: Eva Lozano Montero.

\section{CONCLUSIONES}

Los enfoques diversos que sostienen las disciplinas de ingeniería de costos y contabilidad de costos, en cuanto a la clasificación de los elementos del costo de obra, ya sean directos e indirectos, provocan confusión y representan barreras para su interpretación. Ambas disciplinas argumentan la razonabilidad de conceptualización y clasificación de los conceptos de obra, lo cierto es que se necesita un entendimiento entre ambas disciplinas, en donde antes de mencionar si un costo es directo o indirecto se analice su incidencia en el contrato de obra, en donde no solamente se aplique una fórmula de manera sistemática, sino que se estudie su relación con la obra.

Los costos indirectos para la industria de la construcción, que en una gran porción son gastos generales; de acuerdo al art. 61 del Reglamento de la Ley de Obra Pública y Servicios Relacionados con la misma para el Estado y los Municipios de Guanajuato, se representan como un porcentaje de los costos directos; si se reclasificaran, cambiaría la decisión tanto del contratista como del contratante. De aquí que el contratista trataría de ocupar solamente al personal administrativo y técnico necesario para la obra, haciendo cada vez más flexible al personal que ocupa, cambiando de costos fijos a costos variables, solicitando asesoría contable y administrativa que lo apoyara en la coordinación del personal a ocupar dependiendo del tamaño de la obra a ejecutar. De acuerdo al criterio de Pareto el contratista habrá de concentrarse en determinar el tamaño de las oficinas de campo y central que sea justamente necesario para la obra a ejecutar. Se hace referencia al criterio paretiano debido al economista Vilfredo Pareto, que establece que el $80 \%$ del esfuerzo es usado en el 20\% del trabajo, también es conocido como la regla 80-20. En los costos de construcción se tiene que un $20 \%$ de los conceptos de trabajo representan el $80 \%$ del importe de obra total (lo relevante), mientras que el $80 \%$ de los ítems más triviales sólo aporta el $20 \%$.

El técnico a través del tiempo, con acopio de información y dedicación, podrá adquirir experiencia, desarrollando su eficiencia, siendo capaz de detectar los elementos, materiales y factores relevantes para la ejecución de la obra.

El contador pasaría de ser un trabajador externo, en donde se enfocaba solamente al pago de impuestos; a ser un asesor permanente en la empresa, en donde el contratista consideraría sus honorarios como una inversión. 
El contratante, al analizar los costos directos e indirectos, espera que los gastos generales (indirectos) sean los mínimos necesarios para ejecutar la obra, el artículo 61 en mención, tiene la finalidad de observar la porción de indirectos respecto a los costos directos, pero si la clasificación no es la más razonable, implica toma de decisiones equívocas e inclusive adjudicación de contratos que no son los más viables.

En realidad no se está a favor de alguna disciplina, lo que se propone es que se entiendan ambas partes y que se unifiquen criterios en beneficio del desarrollo de la empresa, exhortando a que ambas disciplinas visionen a la empresa con un enfoque holístico, derribando la barreras de entendimiento y donde predomine la lógica, razonabilidad y ética profesional. Cada profesionista se dedica a cumplir con las obligaciones propias de su campo laboral, olvidándose de comunicar los problemas comunes de la empresa y proponer soluciones de cooperación mutua.

Este artículo pone de manifiesto la necesidad de reformular lo señalado en el Reglamento de la Ley de Obra Pública y Servicios Relacionados con la misma para el Estado y los Municipios de Guanajuato, en relación con la determinación de los precios unitarios, de forma coordinada con lo establecido en las Normas de Información Financiera; para lo cual se hace necesario que la Comisión de Obra Pública del Congreso trabaje de manera conjunta con el Consejo Mexicano para la Investigación y Desarrollo de Normas de Información Financiera (CINIF) y con el Instituto Mexicano de Contadores Públicos (IMCP), para homologar criterios encaminados hacia la eficiente determinación de los costos unitarios de la obra pública en el Estado de Guanajuato.

Está demostrado que es urgente mejorar el desarrollo de las empresas constructoras, incrementando su competitividad a través de soluciones innovadoras, entre las cuales se proponen las siguientes:

a) Unificar criterios en el análisis y clasificación de costos a través de la Comisión de Obra Pública del Congreso y el CINIF e IMCP.

b) Integrar grupos multidisciplinarios.

c) Por lo anterior, es necesario retroalimentar los acuerdos obtenidos por medio de los emisores de la normatividad, realizando reuniones con representantes de la Secretaría de Obra Pública (SOP), Colegios de Ingenieros Civiles y Arquitectos, Colegios de Contadores Públicos, representantes de la Cámara Mexicana de la Industria de la Construcción (CMIC), entre otros; en donde se expongan motivos y lo más relevante, la unificación de criterios de clasificación.

d) Impulsar la iniciativa y asesoría del contador o administración en coordinación con el ingeniero civil o arquitecto, reflejando un mejor entendimiento y coadyuvando a la eficiente toma de decisiones.

e) Apoyar las adecuaciones que sean necesarias beneficiando la competitividad de las empresas.

BIBLIOGRAFÍA

ARBOLEDA LÓPEZ, Sergio Andrés, Presupuesto y programación de obras civiles. México, D. F. Editorial Instituto Tecnológico Metropolitano, 2007, p. 84. 
GARCÍA COLÍN, Juan, Contabilidad de $\operatorname{costos} 2$, segunda edición, México, D. F., ed. Mc Graw Hill, 2001, p. 13.

GONZÁLEZ MELÉNDEZ, Raúl, “Unificando Criterios”, Ingeniería Financiera y de Costos, México, D.F. año LIX, núm., 484, agosto de 2009, pp. 28-32.

RAMÍREZ PADILLA, David Noel, Contabilidad administrativa, octava edición, México, D.F., ed. McGraw Hill, 2008, p. 37.

SUÁREZ SALAZAR, Carlos, Costo y tiempo en edificación, $3^{\text {a }}$. Edición, México, D.F., Editorial Limusa, 2005, p. 25.

VARELA ALONSO, Leopoldo, Ingeniería de costos, teoría y práctica en construcción, México, D.F., editorial InterCost, 2009, p. 129.

\section{DOCUMENTOS}

Periódico oficial del gobierno del Estado. Reglamento de la ley de obra pública y servicios relacionados con la misma para el Estado y los municipios de Guanajuato, Guanajuato, Gto. 2005, número 148, pp. 32-33.

Normas de información financiera, sexta edición, México, D.F. Instituto Mexicano de Contadores Públicos, 2011, p. 717.

Diario Oficial de la Federación. Reglamento de la Ley de Obras Públicas y Servicios Relacionados con las mismas, 2010, p. 72. 
